\title{
MiR-769-5p inhibits cancer progression in oral squamous cell carcinoma by directly targeting JAK1/STAT3 pathway
}

\author{
Y. ZHOU ${ }^{1}, \mathrm{X} . \mathrm{M} . \mathrm{XU}^{2}$, Y. FENG ${ }^{3, *}$ \\ ${ }^{1}$ Department of Stomatology, Hospital of 363, Chengdu, China; ${ }^{2}$ Department of Orthodontics, Hospital of Stomatology, Southwest Medical Uni- \\ versity, Luzhou, China; ${ }^{3}$ Department of Pediatric Dentistry, Hospital of Stomatology, Southwest Medical University, Luzhou, China \\ *Correspondence: qinnqw@163.com
}

Received July 03, 2019 / Accepted September 11, 2019

\begin{abstract}
Oral squamous cell carcinoma (OSCC) is the most common human malignancy worldwide with a high mortality rate. MiR-769-5p has been reported to be downregulated in tissues and blood of OSCC patients. However, the exact roles and pathogenesis of miR-769-5p involved in OSCC remain unclear. The expressions of miR-769-5p and Janus kinase (JAK1) in OSCC tissues and cells were assessed by RT-qPCR and western blot assay. Expressions of apoptotic-related (Bcl-2, Bax, and cleaved-caspase 3) and EMT-associated proteins (MMP9, E-cadherin, N-cadherin, and Vimentin) were detected by western blot assay. The effect of miR-769-5p and JAK1 on proliferation, migration, invasion, and apoptosis was evaluated by CCK-8, transwell, and flow cytometry assays, respectively. The binding interaction of miR-769-5p and JAK1 were predicted by TargetScan and demonstrated by dual-luciferase reporter assays. The volume and weight of the tumor were measured in the subcutaneous transplantation experiment. MiR-769-5p was downregulated, and JAK1 was upregulated in OSCC tissues and cells. MiR-769-5p restrained Bcl-2, MMP9, N-cadherin, and Vimentin protein level and accelerated Bax, cleaved-caspase 3 and E-cadherin protein level, while JAK1 partly overturned these effects. Also, miR-769-5p suppressed proliferation, migration, invasion, and increased apoptosis of OSCC, while the reintroduction of JAK1 abolished these effects. Moreover, JAK1 was verified to be the target of miR-769-5p. In addition, miR-769-5p inhibited the development of OSCC cells in vivo. These results indicate that miR-769-5p suppressed OSCC cell development via targeting the JAK1/STAT3 pathway, providing an underlying therapeutic method for OSCC.
\end{abstract}

Key words: miR-769-5p, JAK1/ STAT3 pathway, oral squamous cell carcinoma

Oral cavity cancer is the eighth most common malignancy in men, with around 354,864 new cases and 177,384 deaths in 2018 worldwide [1]. Oral squamous cell carcinoma (OSCC) is accounting for $90 \%$ of oral cavity cancer [2]. Environmental and genetic factors have been considered to be involved in the etiology; especially, tobacco and alcohol are the pathogenic risk factors for oral cavity cancer [3]. Despite chemotherapy, radiotherapy and surgery have slightly advanced in treatment modalities, the 5-year survival rates remain less than $50 \%$ after diagnosis for OSCC [4]. For this reason, it is imperative to discover more effective treatments for OSCC.

MicroRNAs (miRNAs) are a class of short, endogenous, non-coding RNAs, which partake in the regulation of protein-coding gene by suppressing translation of mRNAs [5]. In recent years, many miRNAs have been demonstrated to exert the role of oncogenes or tumor inhibitors in various cancers, including OSCC [6]. For instance, Feng et al. proved that miR-22 showed a suppressive role by curbing proliferation, invasion, and migration in oral squamous cell carcinoma development [7]. Hiroki et al. reported that the overexpression of miR-205-5p suppressed the invasion by regulating the TIMP-2 expression in OSCC [8]. MiR-769-5p, a form of mature miR-769, has been shown to be downregulated in tissues and blood of OSCC patients [9]. Previous studies have also implicated that miR-769-5p was associated with tumor pathogenesis, and proved to inhibit cancer progression in nonsmall cell lung cancer [10] and colorectal cancer [11]. However, the exact roles and pathogenesis of miR-769-5p involved in OSCC remain unclear.

The Janus kinase/signal transducer of activators of transcription (JAK/STAT) pathway, a common signal transduction pathway of multiple cytokines, has been reported to participate in various biological and cellular processes, such as cell proliferation, apoptosis, and differentiation [12, 13]. Moreover, abnormal activation of JAK/STAT has vital roles in diverse tumors [14]. Additionally, a previous report 
has presented that $\mathrm{miR}-448$ restrained pancreatic ductal adenocarcinoma development by targeting the JAK1/STAT3 pathway, suggesting a potential correlation between miRNAs and JAK1/STAT3 in tumor [15].

In this study, we aimed to demonstrate that miR-769-5p impede OSCC development via regulating the JAK1/STAT3 pathway, providing a more effective therapeutic target for OSCC.

\section{Patients and methods}

Clinical specimens and cell culture. Oral squamous cell carcinoma tissues $(n=48)$ and adjacent non-tumor tissues were obtained from patients undergoing a surgical operation at the Department of Stomatology, Hospital of 363. This study got the approval of the Department of Stomatology, Hospital of 363 and every patient gave the informed consent.

A normal human oral keratinocyte cell line (NHOK) was obtained from the Chinese Academy of Sciences (Shanghai, China). Human OSCC cell lines (SCC-4, SCC-9, SCC-25, and CAL-27) were purchased from the American Type Culture Collection (ATCC, Manassas, VA, USA). All OSCC cells were maintained in Dulbecco's modified Eagle's medium (DMEM) (Gibco, Grand Island, NY, USA) containing 10\% fetal bovine serum (FBS, Gibco). NHOK was incubated in KeratinocyteSFM medium (Invitrogen, Carlsbad, CA, USA). All cells were grown in a $5 \% \mathrm{CO}_{2}$ incubator at $37^{\circ} \mathrm{C}$.

RNA extraction and quantitative real-time PCR (RT-qPCR). Total RNA was extracted from OSCC cell using TRIzol reagent (Invitrogen) following the manufacturer's protocol. Then, miR-769-5p reverse transcription assays were carried out by looped miRNA RT primer sets (RiboBio, Guangzhou, China) and PrimerScript RT reagent Kit (Takara, Tokyo, Japan). JAK1 was reverse transcribed into cDNA using PrimerScript RT reagent Kit (Takara). Then, the relative expression level of miR-769-5p was analyzed by a miScript SYBR Green PCR kit (Qiagen, Hilden, Germany) and U6 snRNA was acted as the endogenous control. The quantitative analysis of JAK1 mRNA was performed using the SYBR Premix Ex Taq ${ }^{\mathrm{TM}}$ reagent (Takara) with GAPDH as the internal reference. All data were calculated using the $2^{-\Delta \Delta C t}$ method. The primers to amplify, JAK1, GAPDH and U6 were listed as below: JAK1: 5'-TTCTACATGGGGGGATAG-3' (sense), 5'-TAAGATTGGAAACCCTCTAA-3' (antisense); GAPDH: 5'AGAAGGCTGGGGCTCATTTG-3' (sense), 5'-AGGGGCCATCCACAGTCTTC-3' (antisense); U6, 5'-CTCGCTTCGGCAGCACA-3' (sense), 5'-AACGCTTCACGAATTTGCGT-3' (antisense).

Cell transfection. MiR-769-5p mimics and scrambled mimics control (miR-NC), miR-769-5p inhibitor and scrambled inhibitor control (anti-miR-NC) were purchased from RiboBio. JAK1 small interference RNA (si-JAK1) and scrambled si-RNA control (si-NC) were purchased from Dharmacon Research Inc. (Chicago, IL, USA). The cDNA sequence of JAK1 was cloned into pcDNA3.1 empty vector
(Invitrogen) to generate pcDNA3.1-JAK1 (JAK1). All cells were transfected with Lipofectamine 2000 reagent (Invitrogen) based on the instruction of the manufacturer.

Dual-luciferase reporter assay. JAK1 3'UTR sequence containing wild-type or mutant-type miR-769-5p binding sites were amplified and cloned into the psiCHECK-2 firefly luciferase reporter vector (Promega, Madison, WI, USA), named as JAK1-WT and JAK1-MUT reporter plasmids. And then, the plasmids were co-transfected with miR-NC or miR-769-5p mimics into SSC-25 or SCC- 9 cells. At $48 \mathrm{~h}$ after transfection, the luciferase activities were assessed using the dual-luciferase reporter system (Promega) based on the producer's instructions.

Western blot assays. Total protein was extracted from the acquired tissue or cultured cells by Protein Extraction Reagent (Thermo Fisher Scientific, Waltham, MA, USA), then protein $(50 \mu \mathrm{g})$ were separated by $12 \%$ sodium dodecyl sulphate-polyacrylamide gel electrophoresis (SDS-PAGE), and electrophoretically transferred to a polyvinylidene difluoride (PVDF) membrane (Millipore, Bedford, MA, USA). Then, the membrane was blocked in 5\% non-fat milk in TBST (Tris-buffered saline and 1\% Tween 20) for $1 \mathrm{~h}$, followed by incubated with primary antibody against rabbit-anti-JAK1 (1:1000, ab47435, Abcam, Cambridge, MA, USA), Bcl2 (1:1000, no. 3498, Cell Signaling Technology, Beverly, MA, USA), Bax (1:1000, no. 2772, Cell Signaling Technology), pro-caspase-3 (1:1000, ab32150, Abcam), cleaved caspase-3 (1:1000, ab13847, Abcam), STAT3 (1:1000, ab68153, Abcam), p-STAT3 (1:1000, ab32143, Abcam), E-cadherin (1:1000, ab15148, Abcam), Vimentin (1:1000, ab137321, Abcam), N-cadherin (1:1000, ab18203, Abcam), MMP9 (1:1000, ab76003, Abcam) and GAPDH (1:2000, cat. no. ab181602, Abcam) at $4{ }^{\circ} \mathrm{C}$ overnight. Subsequently, the membranes were incubated with horseradish peroxidase (HRP)-linked secondary antibody for $1 \mathrm{~h}$ at room temperature. Protein signal was detected with an enhanced chemiluminescence kit (GE Healthcare UK Ltd, Little Chalfont, UK) and its intensity was analyzed with ImageJ software (NIH, Bethesda, MA, USA).

Tumor xenograft assay. All of the animal experiments were approved by the committee of Animal Research of Department of Stomatology, Hospital of 363. Firstly, pre-miR-769$5 \mathrm{p}$-encoding sequences were subcloned into the Plko-AS2. neo vector (National RNAi Core Facility, Academia Sinica, Taipei, Taiwan). Lentiviral vector was prepared according to standard protocols. Then, $5 \times 10^{6}$ lentiviral-transduced SSC-25 cells were injected subcutaneously into BALB/c nu Neoplasma_2020-03 de mice (5 weeks old). Tumor volume was monitored by a caliper once every 3 days, then tumor volume was calculated using the formula: $\mathrm{V}\left(\mathrm{mm}^{3}\right)=0.5$ $\times$ length $\times$ width $^{2}$. After sacrificing the mice after 18 days, the resected tumor was weighed, followed by the analysis of miR-769-5p expression by quantitative RT-PCR.

Cell viability assay. $2 \times 10^{3}$ transformed cells were seeded into 96-well plates for $0,24,48$ and $72 \mathrm{~h}$ in a humidified air atmosphere containing $5 \% \mathrm{CO}_{2}$ at $37^{\circ} \mathrm{C}$. Afterward, 
$10 \mu \mathrm{l}$ CCK-8 reagent (Beyotime Institute of Biotechnology, Shanghai, China) was added and incubated for $2 \mathrm{~h}$ at $37^{\circ} \mathrm{C}$, following the producer's instructions. Then, the number of cells was assessed using a microplate reader (Bio-Rad, Hercules, CA, USA) at $450 \mathrm{~nm}$.

Cell migration and invasion assay. 24-well Transwell chamber (BD Bioscience, San Jose, CA, USA) was used to assess the cell migration and invasion abilities in OSCC cell lines. For migration assays, $2 \times 10^{5}$ cells were plated in the top chamber of transwells. For invasion assays, $5 \times 10^{4}$ cells were introduced into the top chamber coated with matrigel (BD Bioscience). In both assays, cells in the top chambers were suspended in serum-free medium, and the medium complemented with serum was used as a chemoattractant in the lower chamber. After incubation for $24 \mathrm{~h}$ at $37^{\circ} \mathrm{C}$, cells remaining in the top chamber were removed with a cotton swab. Cells that invaded to the lower surface were fixed and

Table 1. Association of clinicopathological factors with miR-769-5p expression in oral squamous cell carcinoma patients.

\begin{tabular}{lcccc}
\hline \multirow{2}{*}{ Parameters } & Total & \multicolumn{2}{c}{ MiR-769-5p expression } & \\
\cline { 3 - 3 } & & $\begin{array}{c}\text { Low } \\
(\mathbf{n}=\mathbf{2 4})\end{array}$ & $\begin{array}{c}\text { High } \\
(\mathbf{n}=\mathbf{2 4})\end{array}$ & \\
\hline Age & & & & \\
$\quad<60$ & 23 & 11 & 12 & 0.765 \\
$\quad 25$ & 13 & 12 & \\
$\quad$ Gender & & & & \\
$\quad$ Male & 16 & 9 & 7 & 0.426 \\
$\quad$ Female & 32 & 15 & 17 & \\
Tumor size (cm) & & & & \\
$\quad<5$ & 22 & 7 & 15 & $0.014^{*}$ \\
$\quad>5$ & 26 & 17 & 9 & \\
TNM stage & & & & \\
$\quad$ I-II & 36 & 14 & 22 & $0.004^{*}$ \\
$\quad$ III-IV & 12 & 10 & 2 & \\
Lymph node metastasis & & & \\
$\quad$ No & 27 & 11 & 16 & $0.028^{*}$ \\
$\quad$ Yes & 21 & 13 & 8 & \\
\hline
\end{tabular}

Notes: Relative expression of miR-769-5p was calculated using $2^{-\Delta \Delta C t}$ method. Data was shown as mean \pm standard deviation, ${ }^{*} \mathrm{p}<0.05$. stained, then counted and photographed under an inverted microscope.

Cell apoptosis assay. The flow cytometry assay was carried out to measure the effect of miR-769-5p and JAK1 on OSCC cell lines. After $48 \mathrm{~h}$ transfection, cells were stained with the Annexin V-fluorescein isothiocyanate (FITC)/Propidium Iodide (PI) Apoptosis Detection Kit (Invitrogen) according to the producer's instructions. And then, apoptotic cells were detected using the FACScan flow cytometry (BD Bioscience).

Statistical analysis. SPSS 20.0 software (IBM, Chicago, IL, USA) was used to conduct all data, which were presented as mean \pm standard deviation (S.D.). Student's t-test or one-way analysis of variance (ANOVA) was used to contrast the differences of two groups or two more groups. A $\mathrm{p}<0.01$ was manifested the statistically significant.

\section{Results}

MiR-769-5p expression was downregulated in OSCC tissues and cells. First of all, RT-qPCR analysis was employed to assess the expression of miR-769-5p in 48 pairs of OSCC tumor tissues and adjacent normal tissues. As shown in Figure 1A, the miR-769-5p level was obviously decreased in 48 pairs of OSCC tissues in comparison with adjacent normal tissues. Then, we further confirmed that miR-769-5p had lower expression in OSCC cell lines (SSC-25, SCC-4, SCC-9, CAL-27) relative to normal human oral keratinocyte cell line NHOK (Figure 1B). To appraise the prognostic value of the miR-769-5p expression in OSCC, the Kaplan-Meier method and the log-rank test were carried out. As shown in Figure 1C, OSCC patients with low miR-769-5p expression levels had poor overall survival versus those with high miR-769-5p expression levels. Moreover, to explore the interaction of miR-769-5p expression with clinicopathological features, 48 patients with OSCC were analyzed (Table 1). The results displayed that miR-769-5p expression level was positively correlated with tumor size, TNM stages and lymph node metastasis $(\mathrm{p}<0.05)$, while had no correlation with gender and age. These data manifested that miR-769-5p expression was involved in OSCC progression.
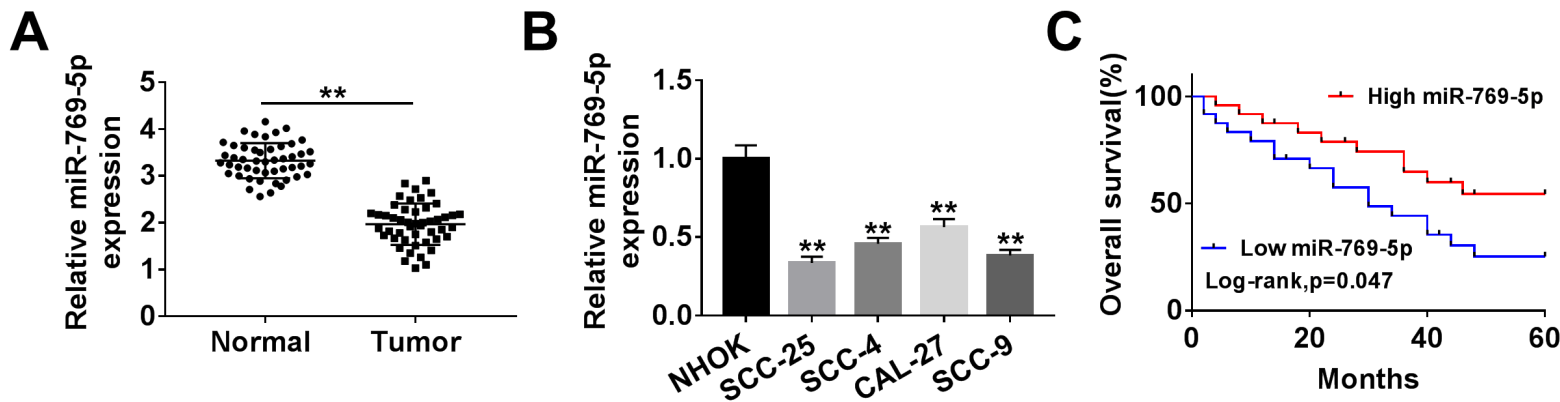

Figure 1. MiR-769-5p expression was downregulated in OSCC tissues and cells. A) RT-qPCR assay was employed to assess the expression profiles of miR-769-5p in 48 pairs of OSCC tissues and adjacent non-tumor samples. B) Expression levels of miR-769-5p in OSCC cell lines (SSC-25, SCC-4, SCC-9, and CAL-27) and normal human oral keratinocyte cell line (NHOK) were measured by RT-qPCR. C) The correlation analysis between miR$769-5 \mathrm{p}$ and the overall survival rate in the OSCC sample. ${ }^{* *} \mathrm{p}<0.01$ 
A

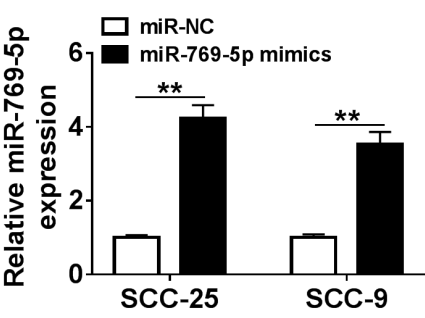

B

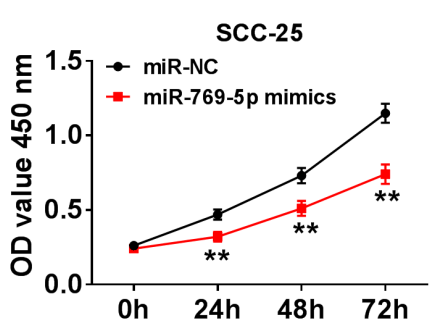

D

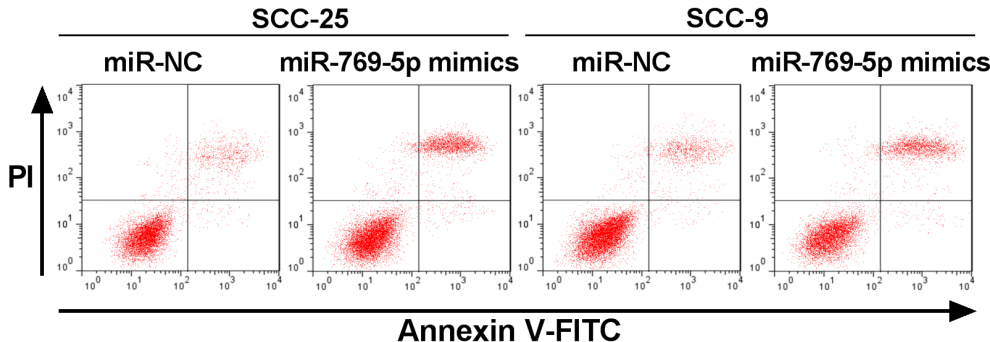

C

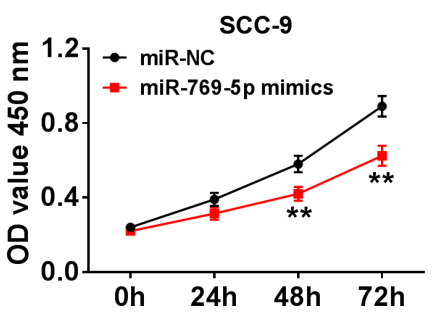

$\mathbf{E}$
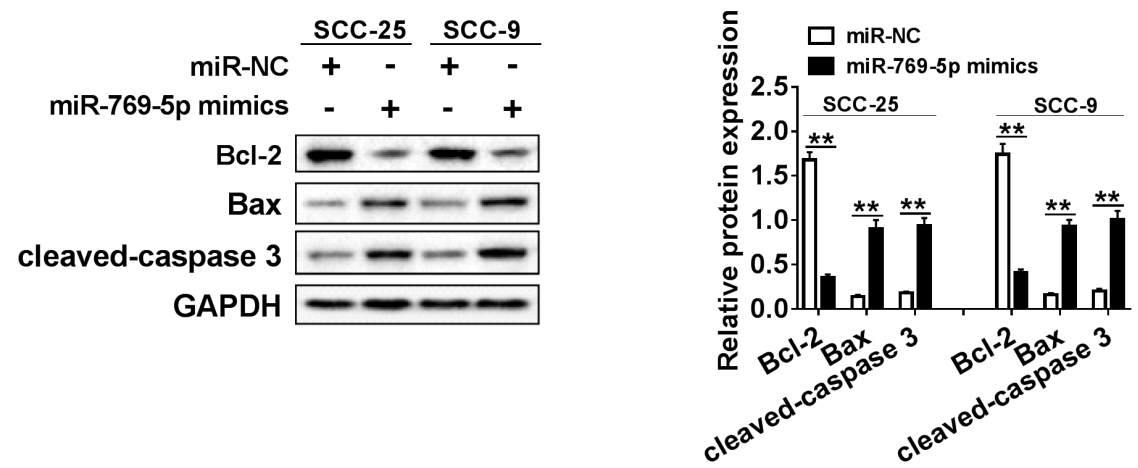

$\mathbf{F}$

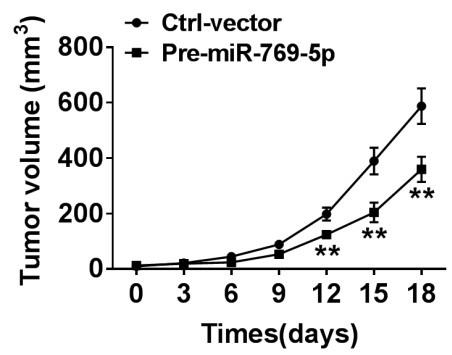

G

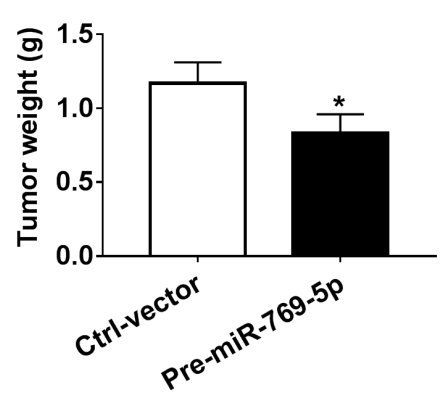

$\mathrm{H}$

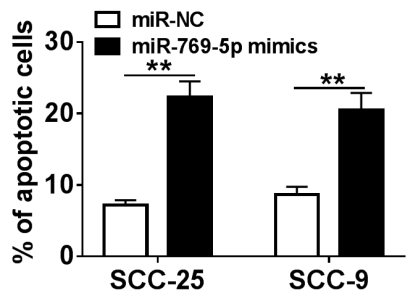

Figure 2. MiR-769-5p suppressed proliferation and induced apoptosis of OSCC cells in vitro. A) Transfection efficiency of the miR-769-5p mimics in SSC-25 and SCC-9 cells. B and C) Cell viability was assessed by CCK-8 assay in SSC-25 and SCC-9 cells transfected with the miR-769-5p mimics. D) Apoptosis rates in miR-769-5p mimics-transfected SSC-25 and SCC-9 cells were assessed by flow cytometry assay. E) Apoptosis-related protein (Bcl-2, Bax and Caspase 3) levels were detected by western blot assay. F and G) Tumor volume and weight of SSC-25 cells transfected with pre-miR-769-5p were measured in vivo. H) MiR-769-5p expression level of SSC-25 cells was determined by RT-qPCR in vivo. ${ }^{* *} \mathrm{p}<0.01$

MiR-769-5p suppressed proliferation and induced apoptosis of OSCC cells in vitro and in vivo. Then, to appraise the function of miR-769-5p in OSCC cells, the overexpression miR-769-5p (miR-769-5p mimics) was performed. The expression level of miR-769-5p was markedly upregulated in OSCC cells transfected with miR-769-5p mimics with respect to OSCC cells with an empty vector (Figure 2A). Thus, we used the overexpression systems to further research the role of miR-769-5p including proliferation and apoptosis. The data displayed that OSCC cell 
proliferation was apparently blocked after introduction with the miR-769-5p mimics (Figures 2B, 2C), while OSCC cell apoptosis was strikingly improved after upregulating the expression of miR-769-5p (Figure 2D). Meantime, knockdown of miR-769-5p promoted cell proliferation, migration, invasion and repressed apoptosis of OSCC cells (Figure S1). To further investigate the effect of the miR-769-5p overexpression on the apoptosis of OSCC cells, the apoptosisrelated protein ( $\mathrm{Bcl}-2$, Bax and Caspase 3 ) activity detection assays were carried out. The data suggested that transfection of the miR-769-5p mimics led to a significant decline in Bax and Caspase 3 activity, and a remarkable increase in the Bcl-2 (B-cell lymphoma/ leukemia) activity (Figure 2E). In addition, to further examine whether miR-769-5p affects the proliferation of OSCC cells in vivo, a subcutaneous xenotransplantation experiment was performed. As shown in Figure 2F and 2G, mice with the miR-769-5p upregulation had a slower decrease in tumor volume and a slower reduc- tion in weight versus controls in vivo. Then, miR-769-5p expression of OSCC cells was obviously accelerated in vivo. In a word, the above data indicated that miR-769-5p could inhibit OSCC progression in vitro and in vivo.

MiR-769-5p suppressed migration and invasion of OSCC cells in vitro. To analyze the effect of miR-769-5p in OSCC cell migration and invasion, the transwell assay was employed. The results of the transwell assays suggested that overexpression of miR-769-5p strikingly curbed the migration and invasion capability of OSCC cells (Figures 3A, 3B). Furthermore, to further disclose the effect of miR-769-5p on the migration and invasion of OSCC cells, epithelialmesenchymal transition (EMT)-related protein (MMP9, E-cadherin, N-cadherin, Vimentin) activity detection assays were carried out. Results indicated that the transfection of the miR-769-5p mimics elicited a notable decline in MMP9, $\mathrm{N}$-cadherin and Vimentin activity, and remarkable enhancement in E-cadherin activity (Figure 3C). Together, these
A
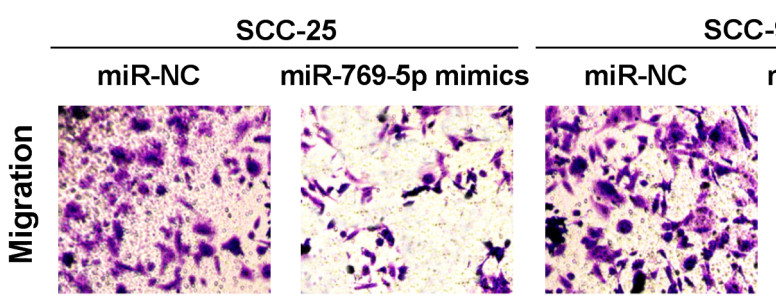

SCC-9 miR-769-5p mimics

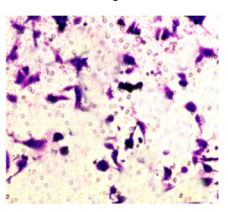

\section{B}

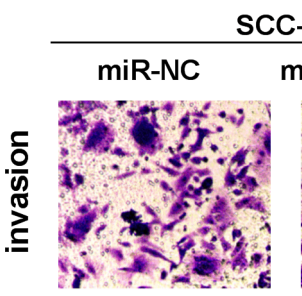

SCC-25 miR-769-5p mimics
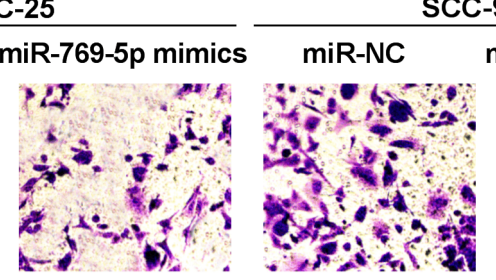

SCC-9 miR-769-5p mimics

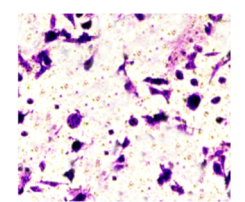

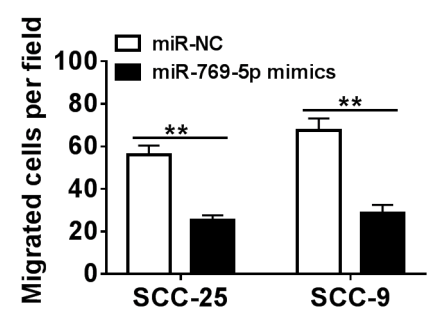

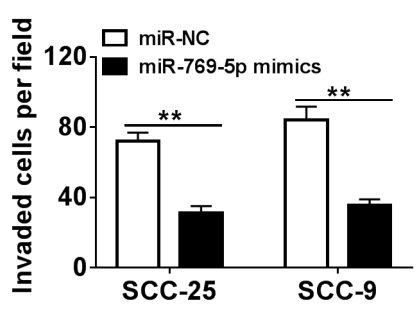

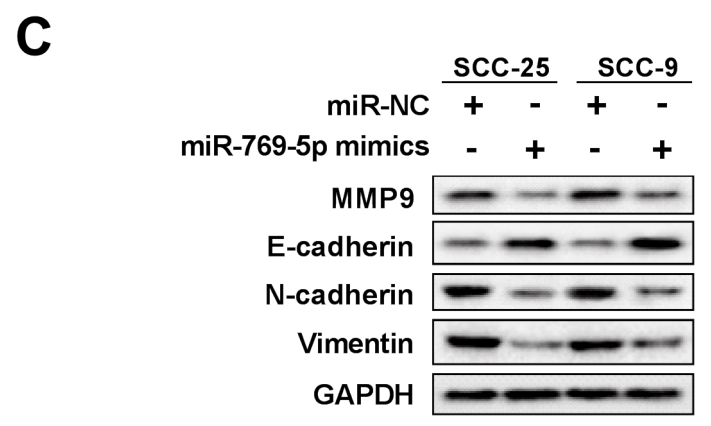

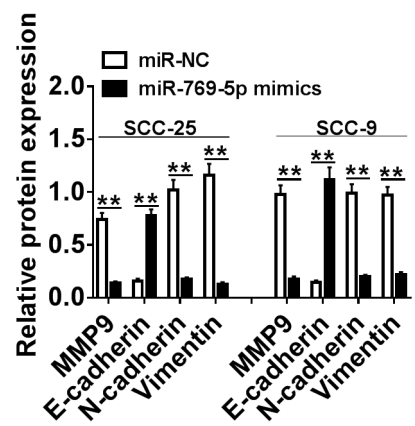

Figure 3. MiR-769-5p suppressed migration and invasion of OSCC cells in vitro. A and B) Migration and invasion abilities in miR-769-5p mimicstransfected SSC-25 and SCC-9 cells were assessed by transwell assay. C) EMT-related protein (MMP9, E-cadherin, N-cadherin, and Vimentin) levels were detected by western blot assay. ${ }^{* *} \mathrm{p}<0.01$ 
A

Position 1255-1261 of JAK1 3'UTR

JAK1-WT 5'...UAGCCUACAUCACCAAGGUCUCU...3'

miR-769-5p 3' UCGAGUCUUGGgUCUCCAGAGU 5'

JAK1-MUT 5'...UAGCCUACAUCACCAGUUGGCAU....3'

D

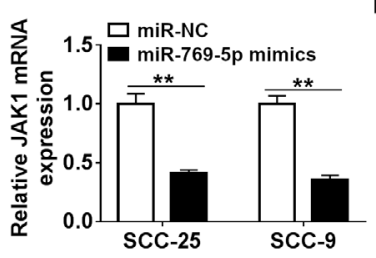

E

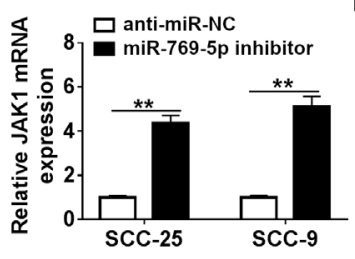

H
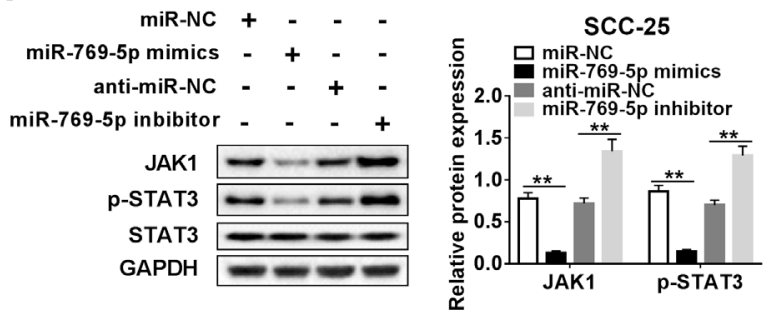

B

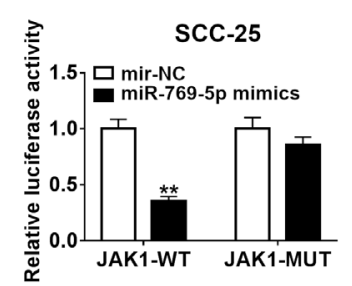

$\mathbf{F}$

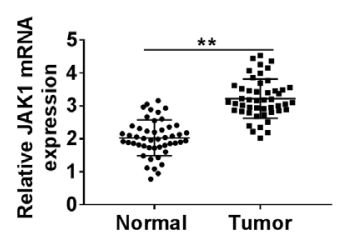

C

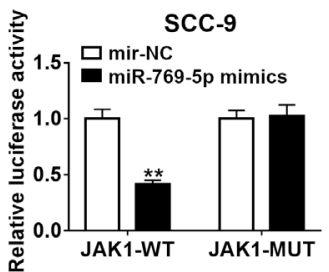

G

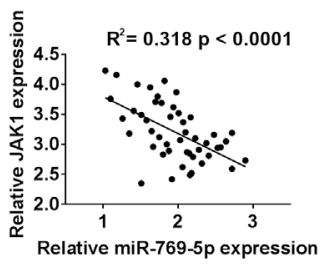

I
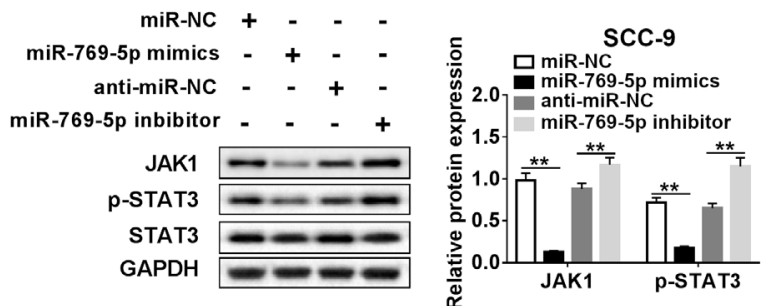

Figure 4. MiR-769-5p-targeted regulation of the JAK1/STAT3 signaling pathway of OSCC cells in vitro. A) Binding sites between miR-769-5p and JAK1 were predicted by TargetScan software. B and C) The effects of the miR-769-5p overexpression on luciferase activity of JAK1-WT or JAK1-MUT reporter were assessed by dual-luciferase reporter assays. D and E) The effect of the miR-769-5p overexpression or knockdown on JAK1 was measured by RT-qPCR in SSC-25 and SCC-9 cells. F) The expression of JAK1 was detected by RT-qPCR in OSCC tissues and adjacent non-tumor samples. E) The correlation coefficient analysis between miR-769-5p and JAK1 was carried out in OSCC tissues H and I). The protein expression of JAK1 and p-STAT3 were determined by western blot analysis in SSC-25 and SCC-9 cells transfected with miR-NC, miR-769-5p mimics, anti-miR-NC, and miR-769-5p inhibitor. ${ }^{*} \mathrm{p}<0.01$

results suggested that miR-769-5p could hinder migration and invasion in OSCC cells.

MiR-769-5p-targeted regulation of the JAK1/STAT3 signaling pathway of OSCC cells in vitro. To explore the mechanisms of miR-769-5p suppressing tumor activity, the TargetScan database was used to predict the potential target of miR-769-5p. The predicted results indicated that miR-769-5p has some binding sites with JAK1 (Figure 4A). To further explore whether the interaction of miR-769-5p with JAK1 was mediated by the binding sites; dual-luciferase reporter assay was employed. Firstly, JAK1-WT or JAK1-MUT reporter vector was cotransfected with miR-NC or miR-769-5p mimics into OSCC cells, followed by the measurement of the luciferase $48 \mathrm{~h}$ after transfection. Results uncovered that the JAK1-WT reporter vector apparently lessened the luciferase activity of miR-769-5p when compared with the JAK1-MUT reporter vector (Figures 4B, 4C). And then, RT-qPCR assay revealed that the miR-769-5p upregulation remarkably blocked JAK1 mRNA expression, whereas the inhibitory effect was disappeared along with miR-769-5p inhibitor (Figures 4D, 4E). To investigate the effect of JAK1 on OSCC tissue, RT-qPCR assay was carried out. As shown in
Figure 4F, JAK1 expression was notably increased in 48 pairs of OSCC tissues in contrast to adjacent non-tumor tissues. Meanwhile, the miR-769-5p expression level was negatively associated with the JAK1 expression level in ccRCC tissues (Figure 4G). Further, we explored whether miR-769-5p could regulate the JAK1/STAT3 signaling pathway, which is allied to tumor growth and metastasis. Western blot results proved that the upregulating of miR-769-5p led to a reduction of JAK1 and p-STAT3 protein expression, while knockdown of miR-769-5p improved the expression of JAK1 and p-STAT3 in SSC-25 and SCC-9 cells (Figures 4H, 4I). Taken together, these data suggested that miR-769-5p could directly regulate the JAK1/STAT3 signaling pathway of OSCC cells in vitro.

MiR-769-5p suppressed proliferation, migration, and invasion of OSCC cells by targeting JAK1 in vitro. To test the mechanisms of miR-769-5p involved in OSCC progression, we executed restoration experiments by transfecting miR-NC, miR-769-5p mimics, miR-769-5p mimics + vector, miR-769-5p mimics + JAK1 into OSCC cells. As exhibited in Figure 5A and 5B, overexpression of miR-769-5p prominently constrained JAK1 or p-STAT3 protein levels of OSCC cells, while rescue experiments of JAK1 evidently abolished 
these effects (Figures 5A, 5B). Then, the JAK1 expression was also measured by RT-qPCR in those transfected cells. Data suggested that overexpression of miR-769-5p remarkably blocked the JAK1 expression level, which was drastically overturned by introducing the JAK1 (Figure 5C). The silencing of JAK1 by si-JAK1 prominently inhibited the expression level of JAK1; miR-769-5p knockdown notably abolished these effects (Figure S2). Furthermore, to observe the effect of miR-769-5p and JAK1 on the apoptosis of OSCC cells, the apoptosis-related protein activity detection assays and flow cytometry assays were performed. The results of detection assays showed that miR-769-5p upregulation distinctly retarded Bcl-2 activity and remarkably increased Bax and cleaved-caspase 3 activities, while transfecting JAK1 substantially abrogated these effect (Figures 5D, 5E). Similar to the detection results, overexpression of miR-769-5p strik- ingly accelerates OSCC cell apoptosis while introduction of the JAK1 effectively blocked OSCC cell apoptosis (Figure 5F). In recent years, some studies manifested that EMT plays important roles in tumor growth, invasion, dissemination, and metastasis [16]. Hence, EMT-related protein (MMP9, E-cadherin, N-cadherin and Vimentin) activity was detected by western blot assay in these transfected cells. Western blot results indicated that the high expression of miR-769-5p improved E-cadherin activity and abated MMP9, N-cadherin and Vimentin activity, and JAK1 reverting attenuated the effect of miR-769-5p overexpression on OSCC cells (Figures 5G, 5H). Then, cell migration, invasion and proliferation, were assessed. Data revealed that miR-769-5p upregulation in OSCC cells obviously repressed migration, invasion and proliferation, nevertheless, revert of JAK1 expression greatly counteracted these effects (Figures 5I-5L). All these
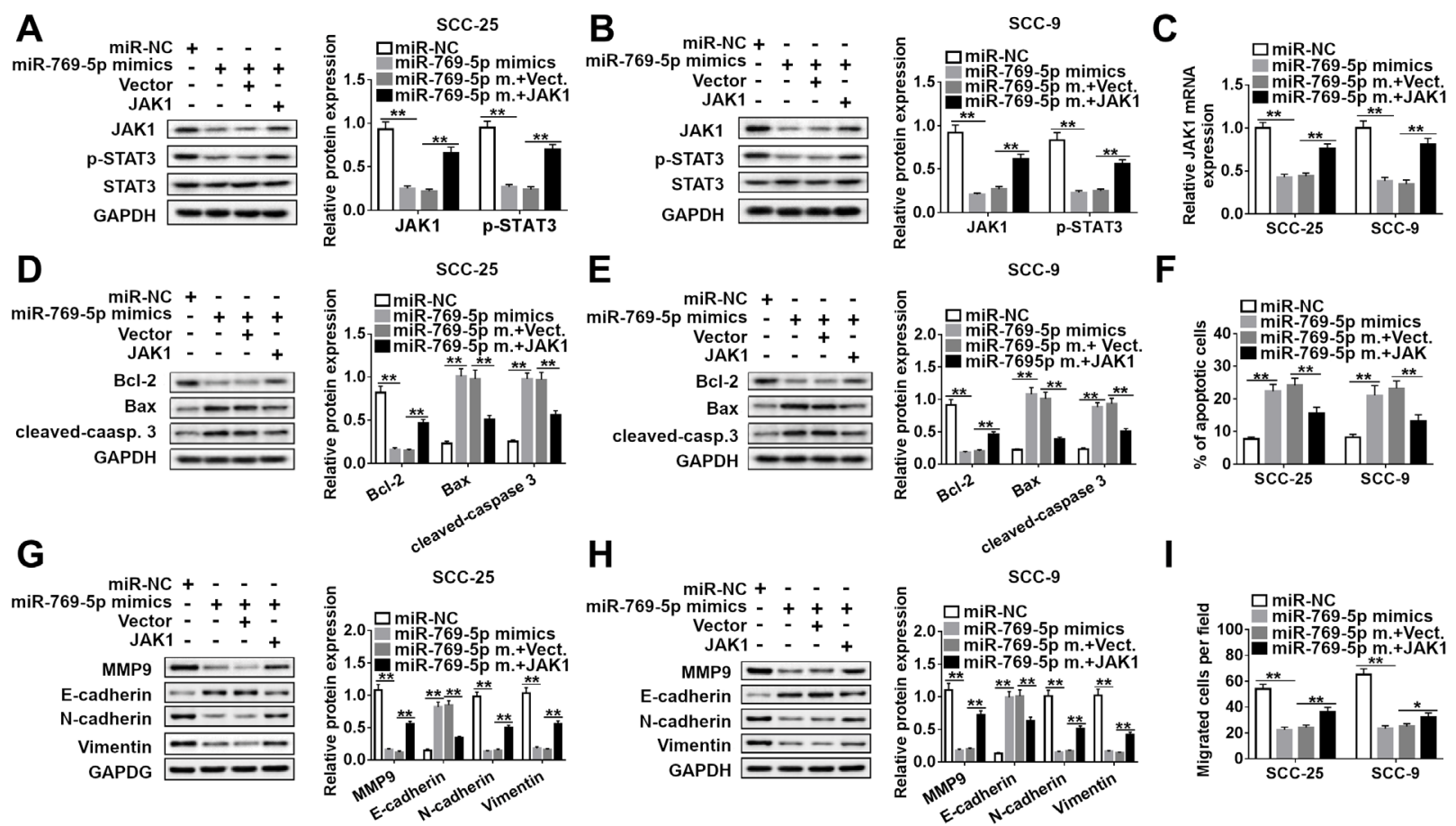

$\mathbf{F}$
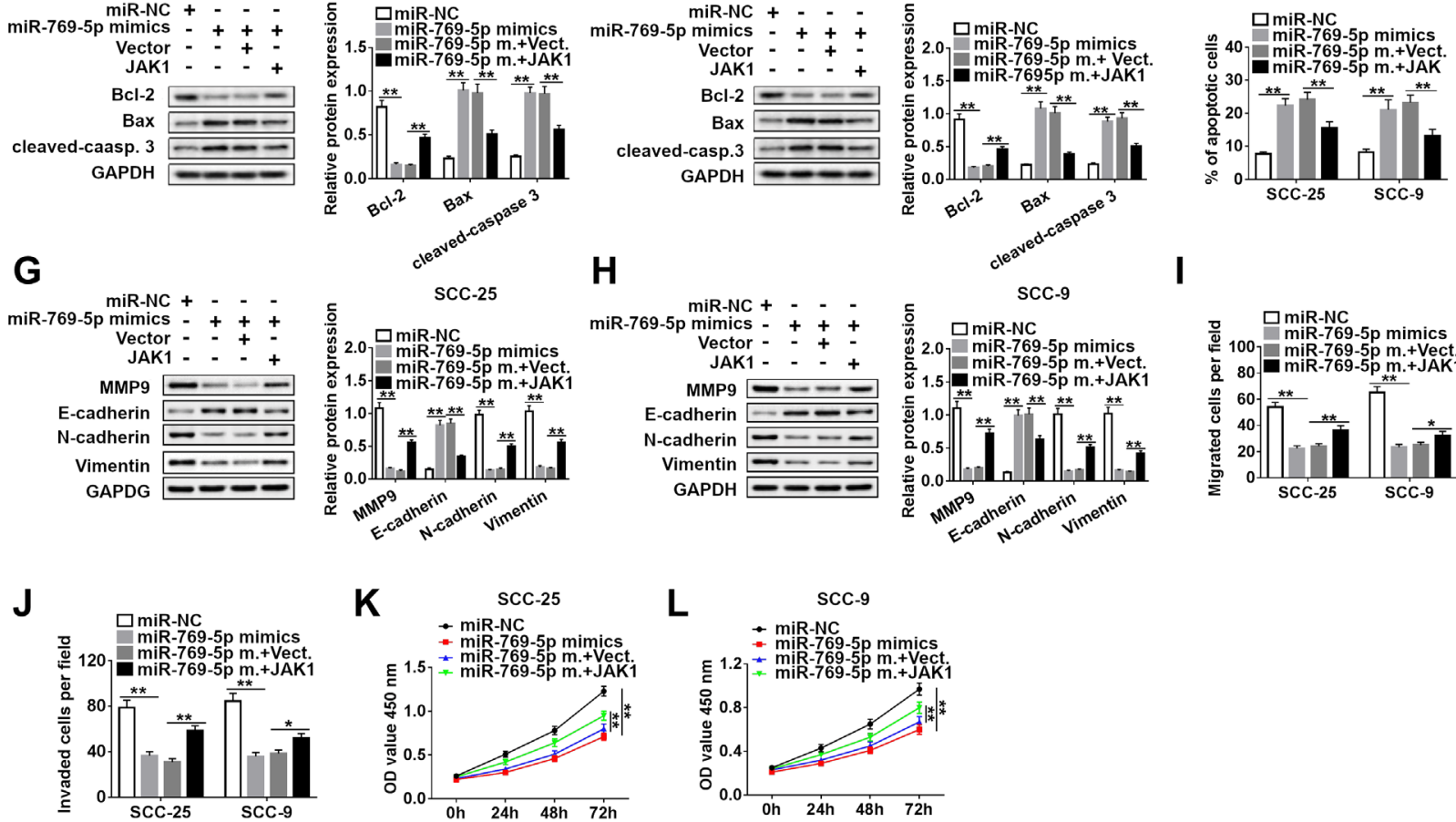

Figure 5. MiR-769-5p suppressed the proliferation, migration, and invasion of OSCC cells by targeting JAK1 in vitro. A and B) JAK1, p-STAT3, STAT3, and GAPDH protein expression were determined by western blot analysis in SSC-25 and SCC-9 cells transfected with miR-NC, miR-769-5p mimics, miR-769-5p mimics + vector, miR-769-5p mimics + JAK1. C) The expression of JAK1 was measured by RT-qPCR assay in transfected SSC-25 and SCC-9 cells. D and E) Apoptosis-related protein (Bcl-2, Bax, and Caspase 3) levels were assessed by western blot analysis in transfected SSC-25 and SCC-9 cells. F) Apoptosis rates were assessed by flow cytometry assay in transfected SSC-25 and SCC-9 cells. G and H) EMT-related protein (MMP9, E-cadherin, N-cadherin, and Vimentin) levels were detected via western blot assay in transfected SSC-25 and SCC-9 cells. I and J) Cell migration and invasion capability were measured by transwell assay in transfected SSC-25 and SCC-9 cells. K and L) Cell proliferation viability was monitored using the CCK-8 assay in transfected SSC-25 and SCC-9 cells. ${ }^{* *} \mathrm{p}<0.01, \mathrm{~m} .=$ mimics, Vect. $=$ Vector, casp. $3=$ caspase 3 
results illustrated that miR-769-5p hampered OSCC development by regulating JAK1.

\section{Discussion}

Accruing researches have indicated that miRNAs are dysregulated expression and can work as diagnostic biomarkers in OSCC [17], and are closely associated with the formation and progression of OSCC [18]. MiR-769-5p, a form of mature miR-769, has been identified to have a suppressive role in nonsmall cell lung cancer and colorectal cancer $[10,11]$. Furthermore, the targets of miR-769 and miR-769-5p such as EGFR, HEY1, CDK1, and GSK3B have been identified in multiple cancers $[10,11,19,20]$. In addition, miR-769-5p was downregulated in tissues and blood of OSCC patients. However, the function of miR-769-5p in OSCC is still unknown.

In our study, the expression level of miR-769-5p was firstly detected in OSCC tumor tissues and cells. Results displayed that miR-769-5p expression was evidently downregulated in OSCC tumor tissues and cell lines in comparison with respective control groups. Then, we analyzed the prognostic value and clinicopathology of OSCC. Data analysis indicated that patients with low expression of miR-769-5p had a markedly poor overall survival rate when compared with those patients with high expression of miR-769-5p, meantime; low expression of miR-769-5p is interrelated with tumor size, TNM stages, and lymph node metastasis.

Then, the effect of miR-769-5p on proliferation, apoptosis, migration, and invasion of OSCC cells was measured. The results suggested that the miR-769-5p upregulation constrained the proliferation, invasion, migration, and accelerated apoptosis of OSCC cells. In other words, miR-769-5p as a suppressive factor in the development of OSCC. Simultaneously, downregulation of the protein levels of $\mathrm{Bcl}-2$, MMP9, N-cadherin, and Vimentin, and upregulation of the protein levels of Bax, Caspase 3 and Vimentin also further demonstrated the inhibiting effect of miR-769-5p on OSCC development. Moreover, in vivo, miR-769-5p expression level markedly upregulated in OSCC cells, and miR-769-5p effectively reduced the volume and weight of SSC-25 cells. We first manifested that miR-769-5p acted as a suppressive factor of OSCC cells in vitro and in vivo.

In recent years, mounting evidence suggested that the aberrant activation of the JAK/STAT signaling pathway plays a vital role in the prognosis, pathogenesis, and treatment of solid tumors [21-23]. Studies have demonstrated that miRNAs could target the JAK1/STAT3 signaling pathway, thereby regulating the tumor progression. Yuan et al. reported that miR-340 restrained proliferation and invasion by regulating the JAK1/STAT3 pathway in hepatocellular carcinoma [24]. Yu et al. proved that miR-448 as a tumor suppressor by blocking metastasis of pancreatic ductal adenocarcinoma via the JAK1/STAT3 pathway [15]. JAK1, a member of the JAK family, induced the STAT3 phosphory- lation (p-STAT3) and contributed to the cell development [25]. Thus, JAK1 as a target gene of miR-769-5p in OSCC cells was further found by the TargetScan database analysis and demonstrated by dual-luciferase reporter assays. In our study, we found that JAK1 expression was upregulated and negatively correlated with miR-769-5p expression in OSCC tissues and cells. And we also found that the miR-769-5p overexpression retarded the JAK1 and p-STAT3 expression level, and the miR-769-5p knockdown enhanced the JAK1 and p-STAT3 expression level. Further experiments displayed that the miR-769-5p target regulated the JAK1/ STAT3 signaling pathway of OSCC cells. Thus, we further explored whether the effect of miR-769-5p on OSCC development was mediated by targeting the JAK1/STAT3 signaling pathway. Prior studies have demonstrated that apoptotic-related and EMT-associated proteins are closely correlated with the JAK1/STAT3 pathway $[24,26]$. In our paper, we presented that the overexpression of miR-769-5p apparently downregulated the expression levels of $\mathrm{Bcl}-2$, MMP9, N-cadherin, Vimentin, and evidently upregulated the expression levels of Bax, cleaved-caspase 3, E-cadherin, whereas the restoration of JAK1 expression overturned these effects, suggesting that miR-769-5p regulated the development of OSCC by targeting JAK1. Where after, the function analysis manifested that the upregulation of miR-769-5p blocked the proliferation, migration, invasion, and boosted apoptosis of OSCC cells, while the reintroduction of JAK1 markedly abolished the tumor suppressor effect of miR-769-5p, indicating that miR-769-5p curbed tumor progression possibly via targeting JAK1.

In conclusion, our study revealed that miR-769-5p was low expressed in OSCC tissues and cell lines. Mechanistically, we for the first time demonstrated miR-769-5p suppressed the proliferation, migration, invasion and elevated the apoptosis of OSCC cells via targeting the JAK1/ STAT3 signaling pathway in OSCC. Our findings illuminate an underlying molecular basis of miR-769-5p in OSCC progress and providing a more effective therapeutic method for OSCC.

Supplementary information is available in the online version of the paper.

Acknowledgments: Joint Project of Cangzhou-Southwest Medical University: Youth Innovation Team of Orthodontic Tooth Movement Mechanism NO:2016LZXNYD-T04. Basic Research on the Application of Cangzhou Science and Technology and Talents Bureau NO:2018-JYJ-37.

\section{References}

[1] BRAY F, FERLAY J, SOERJOMATARAM I, SIEGEL RL, TORRE LA et al. Global cancer statistics 2018: GLOBOCAN estimates of incidence and mortality worldwide for 36 cancers in 185 countries. CA Cancer J Clin 2018; 68: 394-424. https://doi.org/10.3322/caac.21492 
[2] RIVERA C. Essentials of oral cancer. Int J Clin Exp Pathol 2015; 8: 11884-11894.

[3] GHANTOUS Y, SCHUSSEL JL, BRAIT M. Tobacco and alcohol-induced epigenetic changes in oral carcinoma. Curr Opin Oncol 2018; 30: 152-158. https://doi.org/10.1097/ CCO.0000000000000444

[4] SIEGEL R, MA J, ZOU Z, JEMAL A. Cancer statistics, 2014. CA Cancer J Clin 2014; 64: 9-29. https://doi.org/10.3322/ caac. 21208

[5] BARTEL DP. Metazoan MicroRNAs. Cell 2018; 173: 20-51. https://doi.org/10.1016/j.cell.2018.03.006

[6] CAI Z, HAO XY, LIU FX. MicroRNA-186 serves as a tumor suppressor in oral squamous cell carcinoma by negatively regulating the protein tyrosine phosphatase SHP2 expression. Arch Oral Biol 2018; 89: 20-25. https://doi. org/10.1016/j.archoralbio.2018.01.016

[7] FENG X, LUO Q, WANG H, ZHANG H, CHEN F. MicroRNA-22 suppresses cell proliferation, migration and invasion in oral squamous cell carcinoma by targeting J Cell Physiol 2018; 233: 6705-6713. https://doi.org/10.1002/jcp.26331

[8] NAGAI H, HASEGAWA S, UCHIDA F, TERABE T, ISHIBASHI KANNO N et al. MicroRNA-205-5p suppresses the invasiveness of oral squamous cell carcinoma by inhibiting TIMP2 expression. Int J Oncol. 2018 ; 52: 841-850. https:// doi.org/10.3892/ijo.2018.4260

[9] PEDERSEN NJ, JENSEN DH, LELKAITIS G, KISS K, CHARABI BW et al. MicroRNA-based classifiers for diagnosis of oral cavity squamous cell carcinoma in tissue and plasma. Oral Oncol 2018; 83: 46-52. https://doi.org/10.1016/j. oraloncology.2018.05.020

[10] MA G, ZHU J, LIU F, YANG Y. Long Noncoding RNA LINC00460 Promotes the Gefitinib Resistance of Nonsmall Cell Lung Cancer Through Epidermal Growth Factor Receptor by Sponging miR-769-5p. DNA Cell Biol 2019; 38: 176183. https://doi.org/10.1089/dna.2018.4462

[11] WANG L, XU M, LU P, ZHOU F. microRNA-769 is downregulated in colorectal cancer and inhibits cancer progression by directly targeting cyclin-dependent kinase 1 . Onco Targets Ther 2018; 11: 9013-9025. https://doi.org/10.2147/ OTT.S183847

[12] O'SHEA JJ, SCHWARTZ DM, VILLARINO AV, GADINA M, MCINNES IB et al. The JAK-STAT pathway: impact on human disease and therapeutic intervention. Annu Rev Med 2015; 66: 311-328. https://doi.org/10.1146/annurevmed-051113-024537

[13] ZHANG JR, ZHU RH, HAN XP. MiR-410 affects the proliferation and apoptosis of lung cancer A549 cells through regulation of SOCS3/JAK-STAT signaling pathway. Eur Rev Med Pharmacol Sci 2018; 22: 5994-6001. https://doi. org/10.26355/eurrev_201809_15934

[14] PENCIK J, PHAM HT, SCHMOELLERL J, JAVAHERI T, SCHLEDERER $M$ et al. JAK-STAT signaling in cancer: From cytokines to non-coding genome. Cytokine 2016; 87: 26-36. https://doi.org/10.1016/j.cyto.2016.06.017
[15] YU DL, ZHANG T, WU K, LI Y, WANG J et al. MicroRNA-448 suppresses metastasis of pancreatic ductal adenocarcinoma through targeting JAK1/STAT3 pathway. Oncol Rep 2017; 38: 1075-1082. https://doi.org/10.3892/or.2017.5781

[16] BRABLETZ T, KALLURI R, NIETO MA, WEINBERG RA. EMT in cancer. Nat Rev Cancer 2018; 18: 128-134. https:// doi.org/10.1038/nrc.2017.118

[17] MANIKANDAN M, DEVA MAGENDHRA RAO AK, ARUNKUMAR G, MANICKAVASAGAM M, RAJKUMAR KS et al. Oral squamous cell carcinoma: microRNA expression profiling and integrative analyses for elucidation of tumourigenesis mechanism. Mol Cancer 2016; 15: 28. https:// doi.org/10.1186/s12943-016-0512-8

[18] SUN CC, ZHANG L, LI G, LI SJ, CHEN ZL et al. The lncRNA PDIA3P Interacts with miR-185-5p to Modulate Oral Squamous Cell Carcinoma Progression by Targeting Cyclin D2. Mol Ther Nucleic Acids 2017; 9: 100-110. https://doi. org/10.1016/j.omtn.2017.08.015

[19] HAN C, SONG Y, LIAN C. MiR-769 Inhibits Colorectal Cancer Cell Proliferation and Invasion by Targeting HEY1. Med Sci Monit 2018; 24: 9232-9239. https://doi.org/10.12659/ MSM.911663

[20] QIU HJ, LU XH, YANG SS, WENG CY, ZHANG EK et al. MiR-769 promoted cell proliferation in human melanoma by suppressing GSK3B expression. Biomed Pharmacother 2016; 82: 117-123. https://doi.org/10.1016/j.biopha.2016.04.052

[21] THOMAS SJ, SNOWDEN JA, ZEIDLER MP, DANSON SJ. The role of JAK/STAT signalling in the pathogenesis, prognosis and treatment of solid tumours. Br J Cancer 2015; 113: 365-371. https://doi.org/10.1038/bjc.2015.233

[22] VAN DER ZEE M, SACCHETTI A, CANSOY M, JOOSTEN R, TEEUWSSEN M, et al. IL6/JAK1/STAT3 Signaling Blockade in Endometrial Cancer Affects the ALDHhi/CD126+ Stem-like Component and Reduces Tumor Burden. Cancer Res 2015; 75: 3608-3622. https://doi.org/10.1158/0008-5472. CAN-14-2498

[23] TACTACAN CM, PHUA YW, LIU L, ZHANG L, HUMPHREY ES et al. The pseudokinase SgK223 promotes invasion of pancreatic ductal epithelial cells through JAK1/Stat3 signaling. Mol Cancer 2015; 14: 139. https://doi.org/10.1186/ s12943-015-0412-3

[24] YUAN J, JI H, XIAO F, LIN Z, ZHAO X et al. MicroRNA-340 inhibits the proliferation and invasion of hepatocellular carcinoma cells by targeting JAK1. Biochem Biophys Res Commun 2017; 483: 578-584. https://doi.org/10.1016/j. bbrc.2016.12.102

[25] YAN CM, ZHAO YL, CAI HY, MIAO GY, MA W. Blockage of PTPRJ promotes cell growth and resistance to 5-FU through activation of JAK1/STAT3 in the cervical carcinoma cell line C33A. Oncol Rep 2015; 33: 1737-1744. https://doi. org/10.3892/or.2015.3769

[26] KAUSHIK N, KIM MJ, KIM RK, KUMAR KAUSHIK N, SEONG KM et al. Low-dose radiation decreases tumor progression via the inhibition of the JAK1/STAT3 signaling axis in breast cancer cell lines. Sci Rep 2017; 7: 43361. https://doi. org/10.1038/srep43361 


\section{MiR-769-5p inhibits cancer progression in oral squamous cell carcinoma by directly targeting JAK1/STAT3 pathway}

Y. ZHOU ${ }^{1}, \mathrm{X} . \mathrm{M} . \mathrm{XU}^{2}$, Y. FENG 3 ,*

Supplementary Information

A

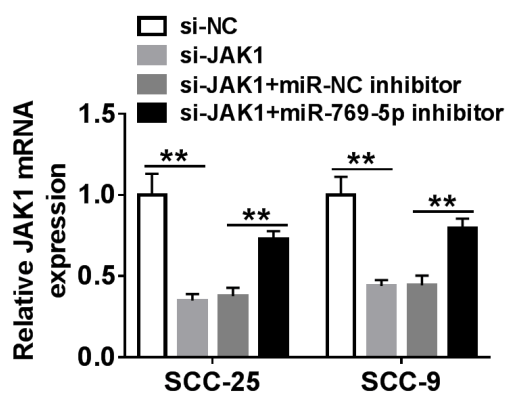

B

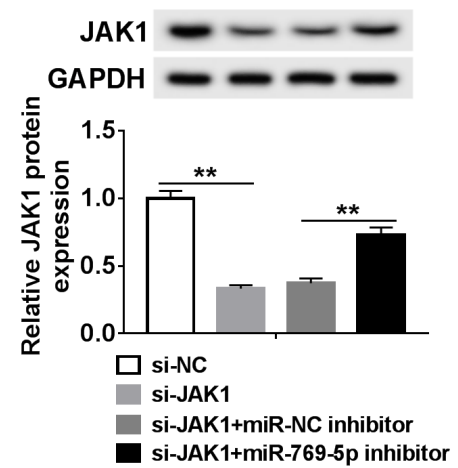

C

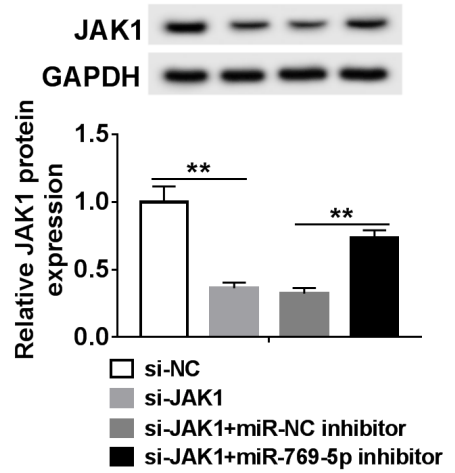

Supplementary Figure 1. Knockdown of miR-769-5p partly abolished the inhibiting effect of si-JAK1 on the JAK1 protein level in in vitro. A) The expression of JAK1 was measured by RT-qPCR assay in SSC-25 and SCC-9 cells transfected with si-NC, si-JAK1, si-JAK1 + miR-NC inhibitor and si-JAK1 + miR-769-5p inhibitor. B and C) The protein level of JAK1 was detected by western blot assay in transfected SSC-25. ${ }^{* *}$ p $<0.01$

A

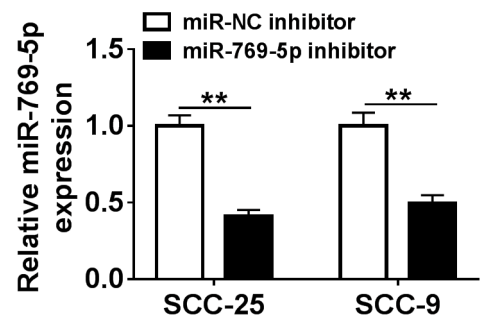

D

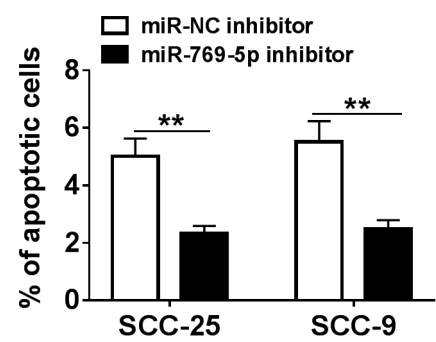

B

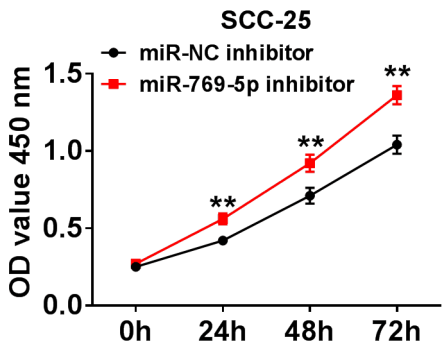

$\mathbf{E}$

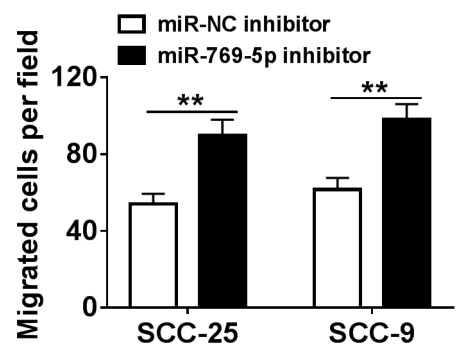

C

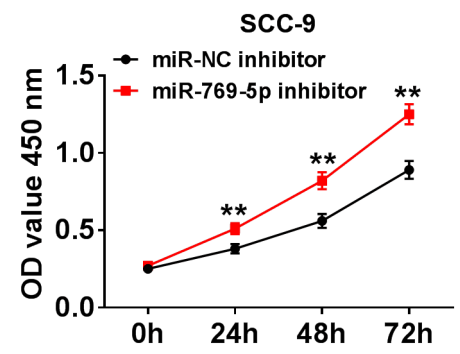

$\mathbf{F}$

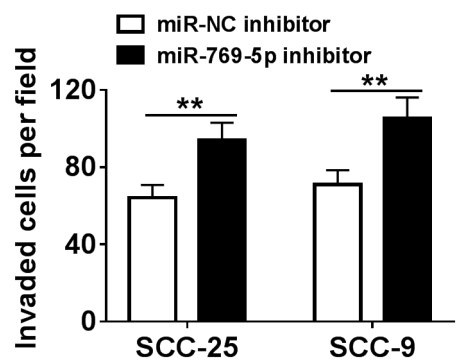

Supplementary Figure 2. Knockdown of miR-769-5p promoted cell proliferation, migration, invasion and repressed apoptosis of OSCC cells. A) The expression of miR-769-5p was measured by RT-qPCR assay in SSC-25 and SCC-9 cells transfected with miR-NC inhibitor and miR-769-5p inhibitor. B and C) Cell proliferation viability was detected by the CCK-8 assay in transfected SSC-25 and SCC-9 cells. D) Apoptosis rates were assessed by flow cytometry assay in transfected SSC-25 and SCC-9 cells. E and F) Cell migration and invasion capability were measured by transwell assay in transfected SSC-25 and SCC-9 cells. ${ }^{* *} \mathrm{p}<0.01$ 